\title{
Molecular imprinting in hydrogels using reversible addition-fragmentation chain transfer polymerization and continuous flow micro-reactor
}

\author{
Porkodi Kadhirvel, ${ }^{a}$ Carla Machado, ${ }^{a}$ Ana Freitas, ${ }^{a}$ Tânia Oliveira, ${ }^{a}$ Rolando \\ CS Dias $^{\mathrm{a}^{*}}$ and Mário RPFN Costa ${ }^{\mathrm{b}}$
}

\begin{abstract}
BACKGROUND: Stimuli responsive imprinted hydrogel micro-particles were prepared using reversible addition-fragmentation chain transfer polymerization for targeting genotoxic impurity aminopyridine in aqueous environment using a continuous flow micro-reactor.

RESULTS: The feasibility of operation with a continuous flow micro-reactor for particles production was demonstrated. A comparative evaluation was carried out between batch and micro-reactor produced imprinted and non-imprinted hydrogels. Experimental results proved that molecular imprints generated by free radical polymerization and controlled radical polymerization showed outstanding performance in adsorption behavior: the $q$ value estimate was about 1000 times higher than the value presented by other researchers. Solid phase extraction results further evidenced the promising imprinting with hydrogels using free radical polymerization and controlled radical polymerization by retaining c. $100 \%$ of 3 -aminopyridine. The imprinting factor of 4.3 presented in this research appears to be the best value shown so far.

CONCLUSION: The imprinted materials were successfully prepared both in batch and with a continuous flow micro-reactor. The inclusion of a reversible addition-fragmentation chain transfer agent in controlled radical polymerization was important in optimizing the experimental conditions in the continuous microfluidic approach. Though the reversible addition-fragmentation chain transfer agent was very useful in controlling the reaction kinetics, imprinted micro-particles showed the existence of both non-specific and imprinted sites. It is worth extending this work to demonstrate the impact of reversible addition-fragmentation chain transfer agents in molecular imprinting, considering also operation in a continuous flow micro-reactor to obtain tailored smart hydrogel particles.
\end{abstract}

(c) 2015 Society of Chemical Industry

Keywords: 3-aminopyridine; smart hydrogel; micro-reactor; molecular imprinting; reversible addition-fragmentation chain transfer; frontal analysis

\section{INTRODUCTION}

Hydrophilic gels of low crosslinked polymer networks swollen in water are for simplicity called hydrogels. ${ }^{1}$ Their ability to absorb a huge amount of water without being dissolved makes those materials useful in medical (artificial cornea implants ${ }^{2}$ ) and tissue engineering (as fillers for soft tissue ${ }^{3}$ and cartilage repairing ${ }^{4}$ ) applications. Stimuli-responsive smart hydrogels (SRSH) have, in addition, the unique capacity of changing volume drastically owing to external stimuli and hence they are extensively used in biotechnology and biomedical fields for drug delivery, enzyme immobilization, tissue culture substrates and as gene carriers. ${ }^{5,6}$ Stimuli (environmental changes) can be $\mathrm{pH}$, temperature, ionic strength, light, electric or magnetic fields. Thermal and $\mathrm{pH}$ responsive smart hydrogels have received more significant interest. ${ }^{7} \mathrm{~N}$-isopropylacrylamide (NIPA), acrylic acid (AA), methacrylic acid (MAA) are a few examples of commonly employed monomers for producing thermal and $\mathrm{pH}$ responsive hydrogels. An overview concerning the applications of stimuli-responsive synthetic materials in medicine can be found in some recent works (e.g. see review paper ${ }^{8}$ and references therein).

Stimuli-responsive smart hydrogels can be obtained conventionally through free radical polymerization (FRP) in a batch reactor, resulting in irregular shaped particles. On the other hand, emulsion, inverse-suspension, precipitation polymerization processes etc., result in mostly uniform spherical shaped particles. Recently, micro-reactors with microfluidic droplet based

\footnotetext{
Correspondence to: Rolando CS Dias, LSRE-Instituto Politécnico de Bragança, Quinta de Santa Apolónia, 5300, Bragança, Portugal. E-mail: rdias@ipb.pt

a LSRE-Instituto Politécnico de Bragança, Quinta de Santa Apolónia, Bragança, Portugal

b LSRE-Faculdade de Engenharia da Universidade do Porto, Rua Roberto Frias $s / n$, Porto, Portugal
} 
techniques have been introduced as an alternative approach to produce continuously spherical/conical particles. ${ }^{9,10}$ Polymerization in continuous flow offers the possibility to increase the throughput at low operating costs, and the resulting polymer is of consistent quality. ${ }^{9-11}$ The continuous flow mixing system allows good control of reagent diffusion, ensuring more defined and reproducible results when compared with batch processes. ${ }^{9-11}$ Production of polymer dispersions with an important impact on process intensification ${ }^{11}$ and continuous synthesis of hollow particles, ${ }^{12}$ are two different examples of recent studies concerning the use of micro-reactors and microfluidics, aiming at increasing the throughput or the creation of particles with special architectures/morphologies. In other recent studies, ${ }^{13}$ microfluidics has also been explored to synthesize different particle morphologies, from nanoscale to microscale, with important applications in controlled drug release (e.g. for cancer therapy).

However, microfluidic devices have their own technical challenges concerning the choice of an optimum residence time, fast enough reaction kinetics, agglomeration of particles and controlling the shape and size of the final product. Controlled radical polymerization (CRP) using a RAFT (reversible addition-fragmentation chain transfer) agent has been considered by several researchers ${ }^{9,14-16}$ to improve the molecular architectures of the resulting materials (aiming at achieving lower chain heterogeneity, controlled topologies, etc.) and to control the polymerization kinetics. Moreover, in the last few years, RAFT polymerization ${ }^{17}$ (and generally $\mathrm{CRP}^{18}$ ) has also been specifically considered for the purpose of producing three-dimensional polymer networks with improved structural homogeneity. Indeed, nowadays, RAFT polymerization is applied in novel polymer syntheses for a diverse range of applications, including the therapeutic and diagnostic fields. ${ }^{19}$

Having considered the benefits of the introduction of a RAFT agent, another goal was added, i.e. to create molecular memory (imprinting) inside the hydrogel network. Imprinting was aimed at creating tailor-made cavities, shaped with a high specificity and affinity towards a target molecule. ${ }^{20-24}$ This has been achieved by introducing the 'target/template' during hydrogel formation. Figure 1 illustrates the three stages of an imprinting process comprising target-monomer self-assembly, polymerization and template removal resulting in the generation of three-dimensional cavities which should ideally match the template molecule in their size, shape and the arrangement of functional groups. Eventually one creates a molecular 'memory' within an imprinted polymer network. Indeed, nowadays, molecularly imprinted polymers (MIPs) have been extensively explored in order to obtain synthetic substitutes for natural antibodies
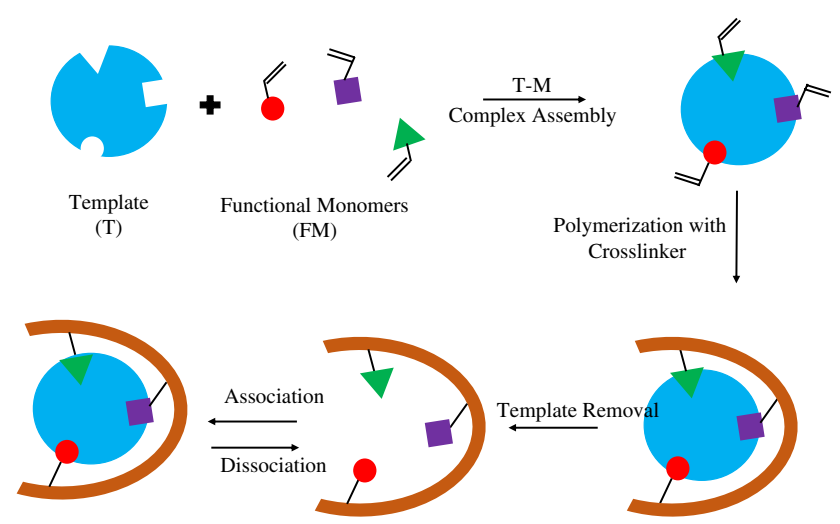

Figure 1. Graphical illustration of molecular imprinting process.

and receptors. ${ }^{25}$ Combination of CRP and molecular imprinting was also recently presented as a route to synthetize advanced MIPs by precipitation polymerization ${ }^{26,27}$ or surface initiated polymerization. ${ }^{28}$ The application of molecular imprinting to produce functional synthetic materials amenable to being stimulated by specific biological or chemical agents has been reviewed elsewhere. ${ }^{29}$

The current work combines three aforementioned lines of research: droplet polymerization to produce hydrogels using microfluidic reactors with controlled radical polymerization (RAFT polymerization is used for improving the molecular architecture of networks) and molecular imprinting. Different polymerization mechanisms (FRP/CRP) were compared with different reactors (conventional batch/micro-reactor) and using imprinting /non-imprinting approaches.

New classes of functional materials with properties such as selective recognition, high capacity, particles in regular shape, controlled swelling degree, smart response to environmental changes and interesting aqueous friendly properties are in increasing demand in the field of biomedical research. Hence, in this approach, the main challenges to be faced were:

1. incorporating molecular memory with an aqueous medium;

2. finding an appropriate crosslinking level lower than $80 \%$ (used in classical MIPs) to $1 \%$ (in hydrogels).

Another concern of this work is the design of tools aiding the synthesis of advanced materials to be applied in a multiplicity of technological/biotechnological fields. For this purpose, the genotoxic impurity 3-aminopyridine (Fig. 2(a)) was considered as a model template to explore the various influencing parameters in obtaining an advanced tailor-made material.<smiles>Nc1cccnc1</smiles>

(a)

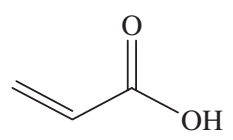

(b)<smiles>Nc1ccncc1</smiles>

(c)

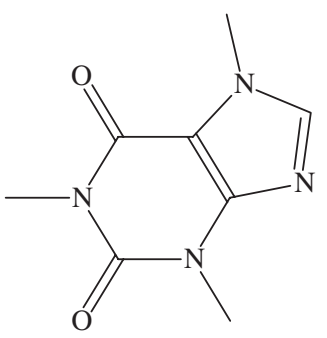

(d)

Figure 2. Chemical structure of model template: (a) 3-aminopyridine (3-APy), functional monomer; (b) acrylic acid (AA), structural analogues; (c) 4-aminopyridine (4-APy); and (d) caffeine (CAF). 


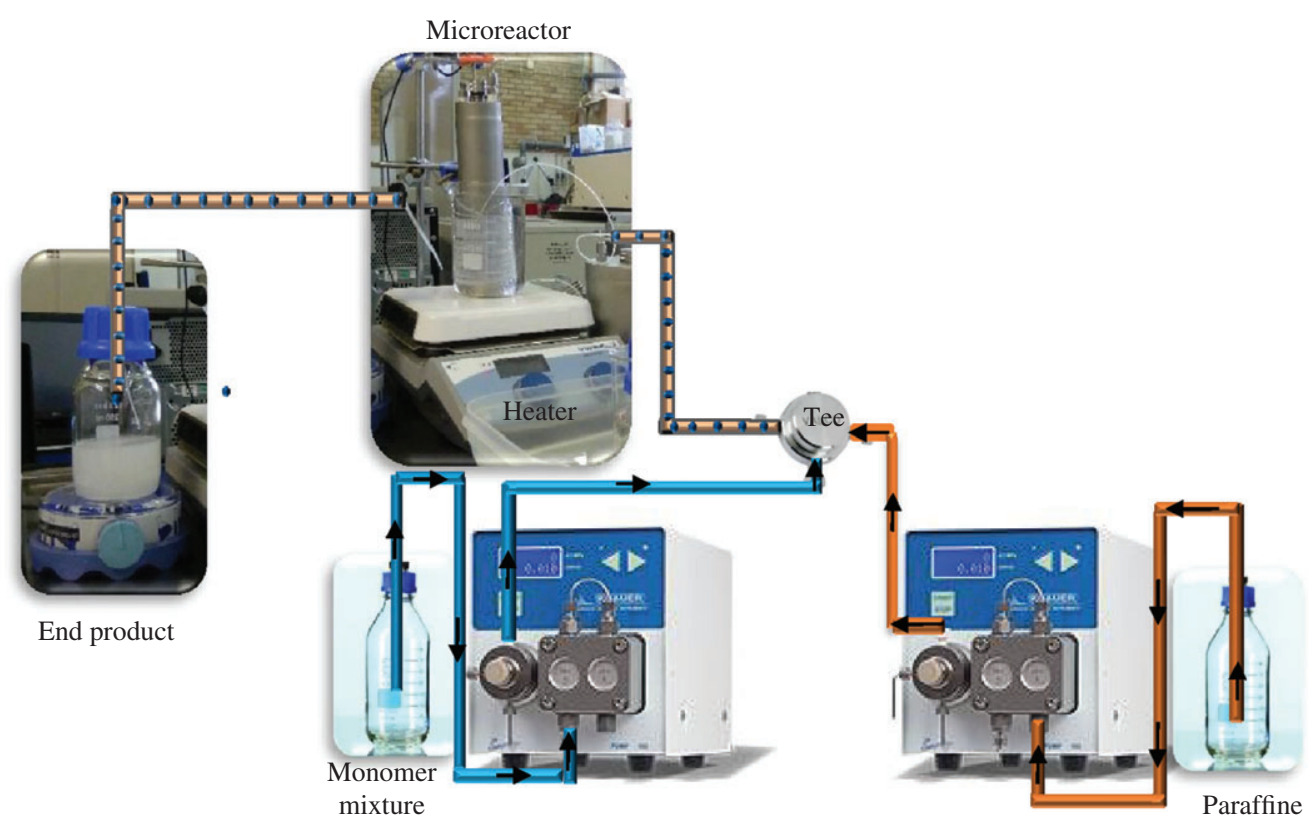

Figure 3. Schematic illustration of the customized micro-reactor used in this work. Through a T connector, two different streams (oil/water phases) are feed to a tubing in an oil bath at controlled temperature. The carrier fluid (e.g. paraffin oil) and the aqueous phase polymer particles (e.g. molecularly imprinted hydrogels) are collected at the outlet.

\section{EXPERIMENTAL}

\section{Materials}

Template 3-aminopyridine (3-APy), functional monomer acrylic acid (AA), crosslinker methylenebisacrylamide (MBAm), initiator V50 (2,2'-azobis (2-methylproprionamidine) dihydrochloride) and RAFT agent 4-cyano-4-(phenylcarbonothioylthio) pentanoic acid (CPA) were purchased from Sigma-Aldrich (Germany). The structural analogue 4-aminopyridine (4-APy) was obtained from Alfa Aesar (Germany). Caffeine (CAF) was procured from Acros Organics (Belgium). Analytical grade acetic acid ( $\mathrm{AcOH})$, methanol $(\mathrm{MeOH})$ and acetone were bought from Fisher Chemical (UK). Paraffin oil was received from Vencilab (Portugal). Millipore water (Milli-Q quality) was used in all the experiments unless otherwise mentioned.

\section{Micro-reactor self-assembly and its operating conditions}

A continuous flow micro-reactor was built using two Knauer HPLC pumps (model Azura P 4. 1S, titanium head) with maximum delivery pressure of $40 \mathrm{MPa}$ and flow rate in the range 0.001 to $10 \mathrm{~mL} \mathrm{~min}^{-1}$. A Valco tee device was used to connect the two lines coming from the pumps with generation of the feed to the micro-reactor. Different T connectors with internal diameters $0.25,0.5,0.75$, and $1.0 \mathrm{~mm}$ were considered. PTFE tubes with different internal diameters $(0.2,0.5,0.8,1.0$ and $1.5 \mathrm{~mm})$ were used as continuous flow micro-reactors. The maximum length of all micro-reactors used was $20 \mathrm{~m}$. The micro-reactor tubing was rolled up on a metallic cylinder and immersed in an oil bath at controlled temperature. A container was connected to the end of the reactor in order to collect the carrier fluid (often liquid paraffin) and the aqueous-phase polymer particles. In this container, a polymer precipitating solvent (methanol or acetone) was included and a magnetic pellet stirred the mixture. This micro-reactor set-up is shown in Fig. 3. This set-up was recently considered by the research team in screening studies concerning the development of tailored imprinted hydrogel particles involving different kinds of templates, monomers and operating conditions. ${ }^{30-32}$

\section{Host-guest interaction}

Interaction between the template and functional monomer (T-M), and the orientation of these molecules were predicted by simple computational simulation. For this purpose the chemical structures of acrylic acid and 3-aminopyridine were drawn in ACS/Chem 3D ultra-software (version 8) and minimized to the lower energy with better orientation by the molecular mechanics (MM) method (minimized energy to minimum RMS gradient of 0.100). Experimentally, a UV-vis spectrometer was used in order to obtain information about the T-M interaction. Blank 3-APy and a mixture of 3-APy and AA solutions were prepared and the absorbance was scanned from 200 to $800 \mathrm{~nm}$. In order to achieve stronger evidence, the template and monomer solutions were prepared in the same molecular ratios as in the pre-polymerization mixture.

\section{Stimuli-responsive smart hydrogels production by free radical polymerization in batch reactor}

In a batch process, the imprinted hydrogel (MIH-FRP) was prepared using 3-APy as template molecule. Acrylic acid (Fig. 2(b)) was used as the main water-soluble functional monomer, methylene bisacrylamide (MBAm) as crosslinker, V50 (2,2'-azobis(2methylproprionamidine) dihydrochloride) was selected as water compatible thermal initiator and water was used as a porogen.

In this typical process, the template 3-APy was mixed first with the functional monomer (AA), crosslinker was added and the content was subsequently dissolved in water. Following that the addition of initiator V50 was made. The solution was transferred to a glass ampoule and purged with a flow of dry argon for 5-10 min. The tube was then sealed and polymerization was started by placing the tubes in a thermostatic oil bath pre-set at $40-50^{\circ} \mathrm{C}$. The hydrogels were removed for further analysis after $24 \mathrm{~h}$. Non-imprinted hydrogel (NIH-FRP) was prepared in parallel to justify the imprinting process in MIH-FRP following the same experimental procedure as for MIH-FRP, eliminating 3-APy. 
Table 1. Pre-polymerization cocktail mixture used for hydrogel synthesis by FRP and CRP

\begin{tabular}{|c|c|c|c|c|c|c|c|c|}
\hline Material & $\begin{array}{l}Y_{M(A A)} \\
\text { (wt \%) }\end{array}$ & $\begin{array}{l}Y_{I(\mathrm{~V} 50)} \\
(\%)\end{array}$ & $\begin{array}{c}Y_{C L}(\text { MBAm) } \\
(\%)\end{array}$ & $\mathrm{Y}_{\mathrm{RAFT}}(\mathrm{CPA}) / /(\mathrm{V} 50)$ & $Y_{T(3-A p y) / C L(M B A m)}$ & $\begin{array}{c}\text { Reaction } \\
\text { temperature }\end{array}$ & $\begin{array}{l}\text { Batch gelation } \\
\text { time, min }\end{array}$ & F.M : C.L \\
\hline MIH-FRP & 11.61 & 0.22 & 1.10 & - & 1 & $50^{\circ} \mathrm{C}$ & 4 & $90: 1$ \\
\hline NIH-FRP & 11.61 & 0.22 & 1.10 & - & - & $50^{\circ} \mathrm{C}$ & 2 & $90: 1$ \\
\hline MIH-CRP-MRP & 40 & 1 & 2 & 0.1 & 1 & $70^{\circ} \mathrm{C}$ & 11 & $49: 1$ \\
\hline NIH-CRP-MRP & 40 & 1 & 2 & 0.1 & - & $70^{\circ} \mathrm{C}$ & 6 & $49: 1$ \\
\hline
\end{tabular}

$\mathrm{Y}_{\mathrm{M}}(\mathrm{wt} \%)$ : Mass fraction of the monomer in the dispersed phase; $\mathrm{Y}_{\mathrm{T} / \mathrm{CL}}$ : mole ratio between template and crosslinker; $\mathrm{Y}_{\mathrm{RAFT} / \mathrm{I}}:$ mole ratio between RAFT and initiator ; $Y_{C L}(\%)$ : mole fraction of crosslinker in the total monomer mixture; $Y_{1}(\%)$ : mole ratio between initiator and monomer.

A summary of the formulations of the imprinted and non-imprinted smart hydrogels is presented in Table 1.

\section{Stimuli-responsive smart hydrogels generation by controlled radical polymerization in a continuous micro-reactor}

Residence time and additional batch reaction time are two important parameters that have to be monitored in micro-reactor microfluidic operation. Residence time can be controlled by flow rate and the polymerization kinetics was observed at small scale before introducing the pre-polymerization cocktail into the micro-reactor assembly. RAFT agent was introduced in the batch protocol to produce imprinted (MIH-CRP) and non-imprinted (NIH-CRP) hydrogels in the micro-reactor by CRP. Table 1 shows the initial composition of the material used for the production of $\mathrm{MIH}-\mathrm{CRP}$ and $\mathrm{NIH}-\mathrm{CRP}$. The well-known drop in polymerization rate associated with $C R P^{17,18}$ when compared with FRP, was also important in order to find more amenable polymerization kinetics allowing the running of the crosslinking process in the continuous flow micro-reactor (e.g. avoiding reactor clogging).

For each CRP SRSH produced in the continuous micro-reactor, the same initial reactant mixture was used to synthesize the corresponding batch material and to estimate the batch gelation time, as detailed in Table 1.

\section{Template removal}

Imprinted and non-imprinted hydrogels prepared either from batch or from the micro-reactor were then isolated and purified through successive cleaning operations. Soxhlet extraction was used in purification procedures to remove specifically the template molecules using $\mathrm{MeOH}$ and acetic acid mixture (90:10). Washing was monitored by UV-vis spectrometer at $231 \mathrm{~nm}$ and was continued until levels of 3-APy became too low to detect.

\section{Stimuli-responsive smart hydrogels characterization}

Hydrogel (dry and swollen state) microscopic images were monitored using a Nikon Microscope ECLIPSE 50i (Japan) and the images were processed by software NIS-Elements version 2.2.1. The swelling ratio (SR) of the batch (FRP) and micro-reactor (CRP) generated hydrogels were also measured by impregnating $5 \mathrm{mg}$ of hydrogel in $10 \mathrm{~mL}$ of $20 \mathrm{mmol} \mathrm{L}^{-1} 3-\mathrm{APy}, 4-\mathrm{APy}$ and CAF for about $24 \mathrm{~h}$ and the mass differences were calculated. Knowing the differences in mass before and after impregnation, SR can be calculated using the following equation:

$$
S R=\frac{\text { mass of swollen polymer }}{\text { mass of dried polymer }}
$$

The effect of drug structure on the affinity and selectivity of the materials was studied by comparing SRs of templates and different chemical structures, namely caffeine and 4-aminopyridine.

Additional features on the morphology of the dried SRSH particles were obtained by SEM. These analyses were performed in the Materials Center of the University of Porto (CEMUP).

Characterization of the dried SRSH products (imprinted/ non-imprinted) was also performed using IR spectroscopy. Particles were crushed, mixed with $\mathrm{KBr}$ and pressed into pellets in order to collect the IR spectra.

\section{Solid phase extraction}

Solid-phase extraction cartridges were packed with $10 \mathrm{mg}$ of the 3-APy imprinted (MIH-FRP and MIH-CRP, batch and continuous micro-reactor synthesized) and the corresponding non-imprinted ( $\mathrm{NIH})$ dry hydrogels. The cartridges were conditioned with $5 \mathrm{~mL}$ of water and loaded with aqueous solution $(2 \mathrm{~mL})$ containing $5 \mathrm{mmol} \mathrm{L}^{-1}$ of 3-APy percolated at a constant flow rate of $0.5 \mathrm{~mL} \mathrm{~min}^{-1}$ in a Visiprep (Supelco, Bellefonte, USA) SPE processing station manifold. After loading, $2 \mathrm{~mL}$ of water was used as the washing solution. The cartridges were thereafter subject to an elution step by percolating $2 \mathrm{~mL}$ of $\mathrm{pH}=2$ and $\mathrm{pH}=8$ aqueous solutions. The elution fractions (loading, washing and elution) from the SPE columns were directly monitored by UV-vis spectrometer with detection performed by UV absorbance at $231 \mathrm{~nm}$. With the absorbance data, the concentration of the bound or adsorbed analytes in the hydrogel was calculated using:

$$
\text { Recovery }(\%)=\frac{[\text { Elute }]}{[\text { Load }]} \times 100
$$

Regeneration of the SPE cartridge was achieved by re-conditioning with $5 \mathrm{~mL}$ of water.

\section{Frontal analysis}

Frontal analysis is a high precision technique for adsorption studies $^{33,34}$ and it was considered here to evaluate the performance of the synthetized smart hydrogels. The studies were performed through packing the materials produced (bulk hydrogels obtained in batch reactor or particles synthetized in micro-reactor) in empty GPC columns with bed lengths/internal diameters $(\mathrm{mm} / \mathrm{mm})$ of $10 / 4.6,33 / 4.6,50 / 4.6$ and 33/8. High swelling of these materials was exploited to induce self-packing of the smart hydrogels in the GPC columns. Analytes saturation and release UV absorbance profiles were monitored continuously in a GPC pumping system by feeding $0.5 \mathrm{mmol} \mathrm{L}^{-1}$ solution of 3-APy (to gain saturation information) and eluted with water (to gain the release profile). 
(a)

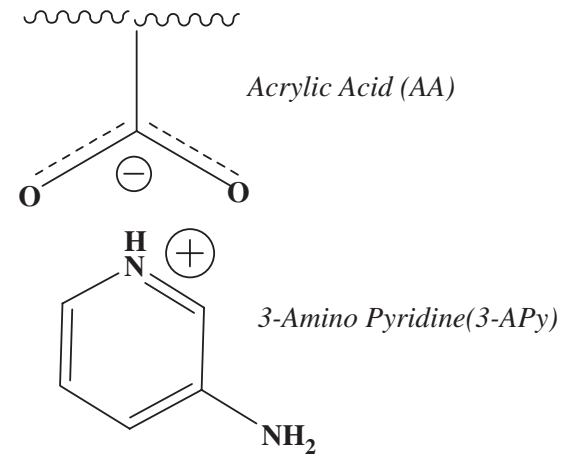

(b)

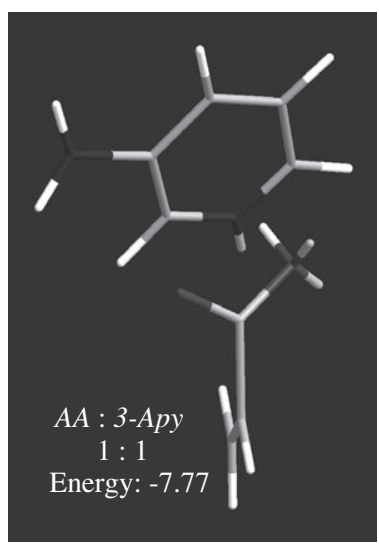

Figure 4. Depiction of the host-guest interaction between the template and the functional monomer (template - monomer self-assembly/T-M): (a) expected T-M orientation; (b) observed T-M orientation by MM energy minimization.

\section{Batch adsorption experiments}

Performance of the synthetized smart hydrogels was also assessed through batch adsorption studies in order to gain insight to the amount of template adsorbed in the hydrogel. A set of $15 \mathrm{~mL}$ capacity centrifuge tubes was used for the batch adsorption experiments. About $10 \mathrm{mg}$ of hydrogel was placed in each centrifuge tube, and this was mixed with $10 \mathrm{~mL}$ of different concentrations of 3-APy and the mixture was equilibrated for about $24 \mathrm{~h}$ under agitation. After equilibration, the supernatant was collected by centrifugation and the concentration measured by UV-vis monitoring.

From the UV-vis absorbance data, the initial and final concentration of the analyte before and after equilibration, $C_{0}$ and $C$, were calculated. The values were substituted in Equation (3) to obtain the adsorbed amount in the hydrogel mass $(\mathrm{g})$. For calculation of the amount of drug adsorbed in the hydrogel (expressed in $\mathrm{mmol}$ of drug per gram of hydrogel) one can use:

$$
q=\frac{V\left(C_{0}-C\right)}{m_{H G}}
$$

where $q$ is the amount adsorbed, $C_{0}$ is the initial and $C$ the equilibrium concentration of the analyte, and $m_{H G}$ is the mass of the hydrogel.

\section{RESULTS AND DISCUSSION}

\section{Host-guest interaction}

A key requirement to achieve promising imprinting inside the hydrogel polymer network is to have a strong interaction between the template and the functional monomer by self-assembly $(T-M)$. The stronger the interaction the better the imprinting efficiency. ${ }^{20,35}$ To detect this T-M orientation, molecular mechanics energy minimization was run using ACS/Chem 3D Ultra. As mentioned earlier, within this research, hydrogels (FRP and CRP) were prepared in aqueous media and to expect promising imprinting, the main prerequisite is to have a better non-covalent interaction, which should be strong enough compared to the hydrogen bonding. It is obvious that in aqueous media, water significantly weakens or completely destroys hydrogen bonding. Therefore, in our work, we were aiming to bring stronger electrostatic interaction between the template and monomer self-assembly (Fig. 4(a)).

The orientation result obtained by molecular mechanics energy minimization is shown in Fig. 4(b) (screen shot) and it is proved

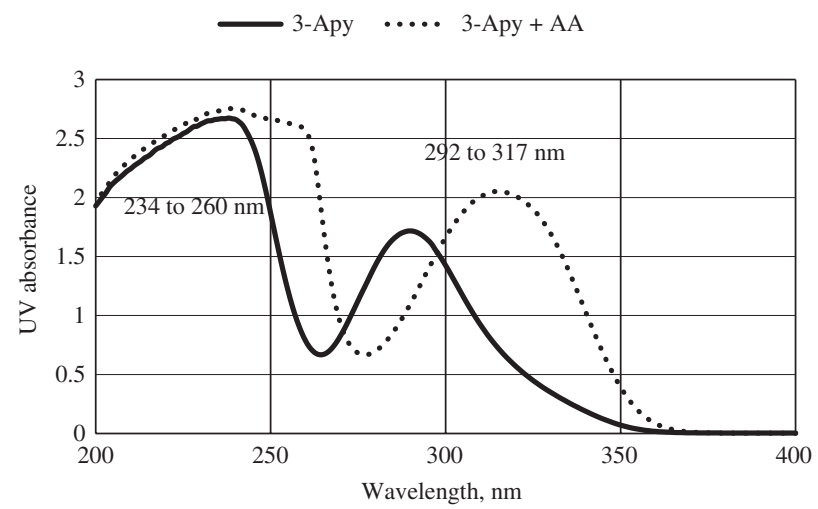

Figure 5. UV-vis spectra of 3-APy and 3-APy $+A A$ aqueous solutions showing absorbance shifts of 234 to $260 \mathrm{~nm}$ and of 292 to $317 \mathrm{~nm}$. Measurements were performed using a $1 \mathrm{wt} \%$ solution of 3-APy in water and a 1 wt $\% 3-A P y+11.61 w t \%$ AA solution of $3-A P y+A A$ in water.

that the 3-APy molecule approaches the AA via electrostatic interaction, as expected.

Further, experimental results of $\mathrm{T}-\mathrm{M}$ interaction monitored by UV-vis spectrometry has been used by several researchers resulting in success. ${ }^{36,37}$ The results of the scanned spectrums are shown in Fig. 5.

Figure 5 clearly shows that the absorption maxima of the 3-APy shifted to higher wavelengths with the addition of functional monomer AA, i.e. from 234 to 260 and from 292 to $317 \mathrm{~nm}$ (red shifted). This red shift behavior in the spectrum after addition of the functional monomer to a fixed concentration of 3-APy in aqueous solution shows that there is formation of a T-M complex by electrostatic interaction. A similar effect has been observed in the case of titration of 2-APy to MAA, where it was found that a strong interaction occurred between aminopyridine and the template molecule. ${ }^{38-40}$

The orientation detection in ACS/3D Chem draw and the absorbance red shift observed by UV-vis experiment confirms the M-T self-assembly by electrostatic interaction and this motivates to proceed with the generation of SRSH hydrogels in aqueous media.

\section{Hydrogel syntheses (FRP and CRP)}

Hydrogel imprints (MIH-FRP) and non-imprinted counterparts (NIH-FRP) were synthesized adopting classical free radical 

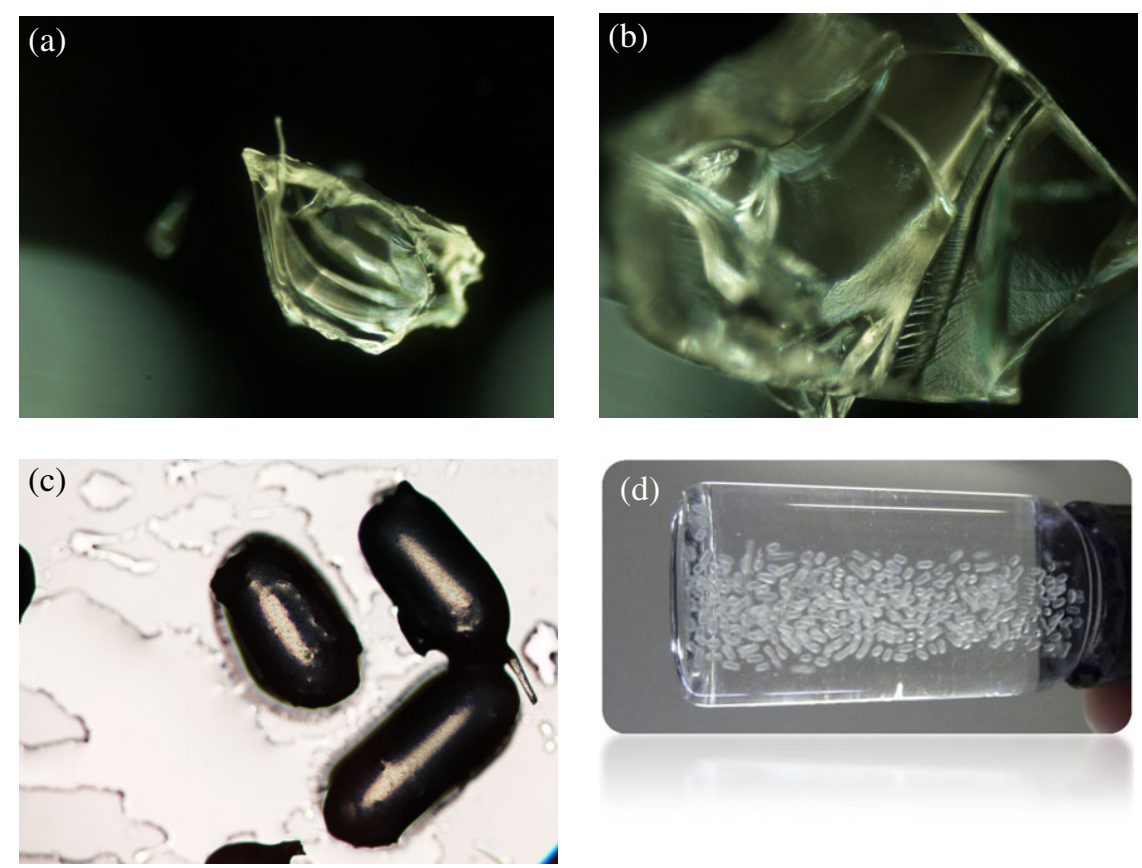

Figure 6. Swollen state images of the hydrogels produced: (a) and (b). Microscopic images of FRP batch produced hydrogels. (c) Microscopic image of AA based RAFT imprinted smart hydrogel (wet) particles obtained in continuous flow micro-reactor, (d) Photograph of AA based RAFT imprinted smart hydrogel particles produced in the micro-reactor.

polymerization as described in the experimental part, while $\mathrm{MIH} / \mathrm{NIH}-\mathrm{CRP}$ microparticles were produced using RAFT agent in the continuous flow micro-reactor (experimental section). Measurement of batch 'gelation time' is a key aspect to assess the feasibility of continuous polymerization with the selected residence time. The residence and the batch reaction times are key parameters influencing microparticle production. As can be seen in Table 1 the reaction time for the MIHs regardless of the polymerization approach (FRP or CRP) is longer (2-fold) when compared with that of the $\mathrm{NIH}$. Apparently the T-M self-assembly in $\mathrm{MIH}$ s caused a longer gelation time. Further it can be seen in Table 1, that the addition of $0.1 \%$ RAFT to the FRP system eventually decreases the reaction rate. In order to achieve optimum conditions for producing microparticle hydrogels in the micro-reactor, the temperature of the reaction was increased from $50^{\circ} \mathrm{C}$ to $70^{\circ} \mathrm{C}$, the former temperature being used in FRP. The hydrogels (MIH-FRP/CRP and NIH-FRP/CRP) synthetized in this way were cleaned in a Soxhlet extractor and the results proved the absence of 3-APy.

\section{Material characterization}

FRP (batch) and CRP (micro-reactor) produced hydrogel (swollen state) images as observed by microscope are shown in Fig. 6(a)-(d). Microscope images presented in Fig. 6 were taken using 40 times magnification, and, according to the instrument calibration ( 1 pixel $=1.684 \mu \mathrm{m}$ ), the width of these images corresponds to $4.3 \mathrm{~mm}$. Photographs of AA based RAFT MIH particles produced in the micro-reactor and presented in Fig. 6(d) were obtained under the following conditions: particles were isolated, dried and poured in aqueous solution with concomitant swelling. The aqueous solution containing the particles is inside a $5 \mathrm{~cm}$ long light-scattering vial (the length of the swollen cylindrical particles is in the range 1 to $2 \mathrm{~mm}$ ).

It can be clearly noticed in Fig. 6 that the micro-reactorgenerated particles are almost uniform and oval in shape whereas the FRP hydrogels produced in the batch reactor are randomly irregularly shaped. Also one can easily observe that the swelling is uniform and controlled in microparticles but not in FRP-produced hydrogels (Fig. 6(a)-(d)). As aimed at in this work we were able to produce SRSH uniformly sized particles which were expected to have better properties, avoiding experimental problems occurring with FRP-produced irregular particles. A few issues to mention are heterogeneous adsorption sites, poor chromatographic properties with low surface area and poor mass transfer, no control in swelling degree, and difficulties leading to high pressure in the chromatographic instruments (exceeding the maximum pressure limit) due to the irregular sized and shaped particles produced by FRP. The particles were of millimeter size range but can be reduced to micrometer range by using lower ID PTFE micro-reactors. This can be explored in future.

The morphology of the different kinds of particles synthetized in this work can be assessed through Fig. 7 where SEM micrographs of imprinted and non-imprinted dried hydrogels produced through FRP and RAFT polymerization are shown.

Figure 8 presents FTIR spectra of the imprinted and non-imprinted dried hydrogels produced through FRP and RAFT polymerization, in batch and continuous flow micro-reactors, as detailed in Table 1. Synthesized particles are very hard, especially those obtained in the continuous flow micro-reactor (higher crosslinker content), thus hampering the formation of pellets with favorable conditions for FTIR analysis (low IR signals were observed). For comparison purposes, the FTIR spectrum of a similar MIH hydrogel obtained through precipitation polymerization was also collected. In this case a soft powder was synthesized and good conditions for FTIR analysis were observed. Note that before drying for the FTIR measurements, all the products were isolated by methanol washing in order to remove unreacted monomers, organic oils, etc. Soxhlet extraction was not considered at this stage in order to try to avoid template removal in the imprinted materials. Comparison of the different spectra shown in Fig. 8 

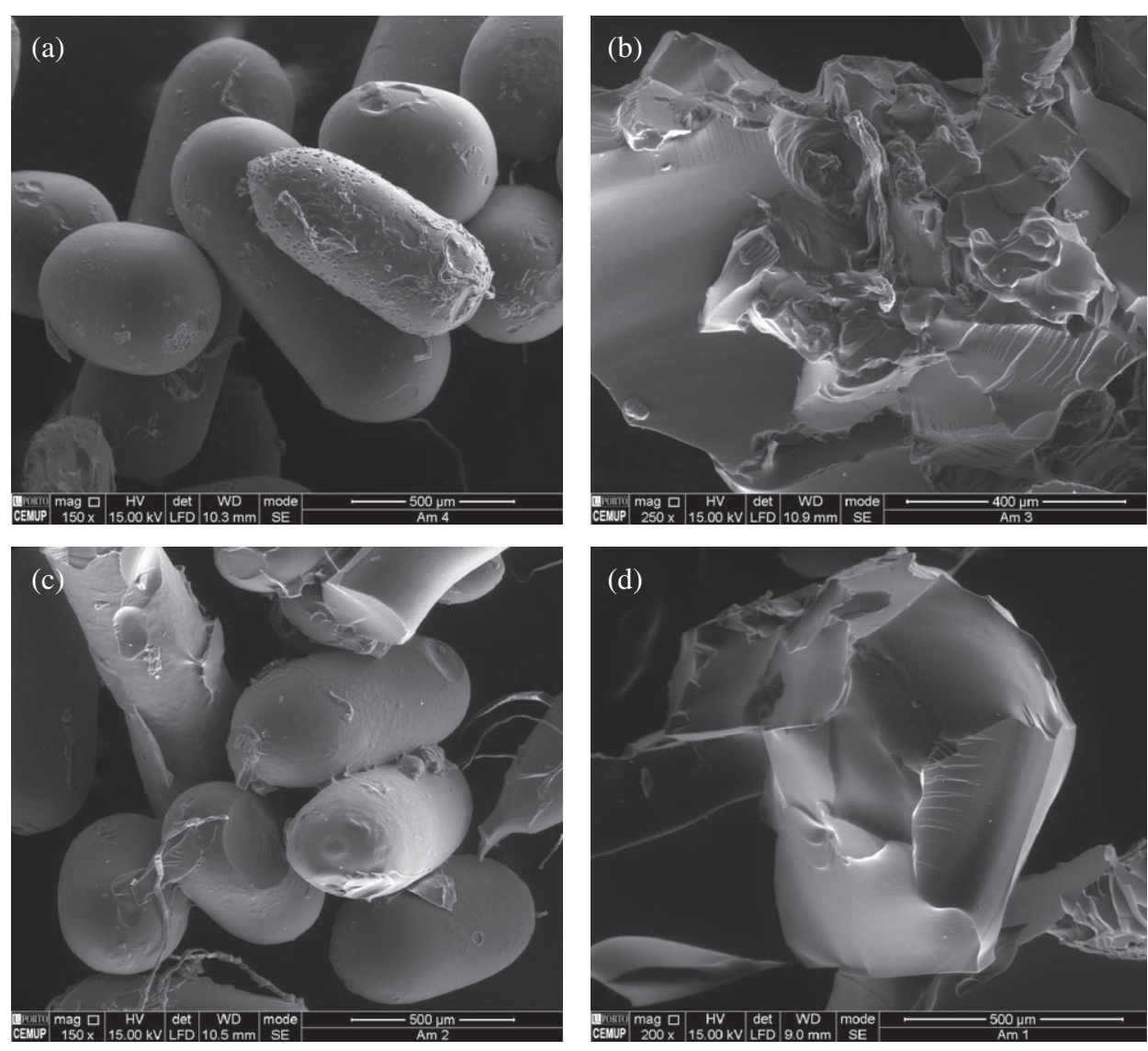

Figure 7. SEM micrographs of imprinted and non-imprinted dried hydrogels produced through FRP and RAFT polymerization. (a) MIH particles synthesized through RAFT polymerization in continuous flow micro-reactor. (b) MIH products obtained through FRP in batch reactor. (c) NIH particles synthesized through RAFT polymerization in continuous flow micro-reactor. (d) NIH products obtained through FRP in batch reactor.

(considering the precipitation polymerization material as reference) seems to indicate that only simple structural features of the synthetized networks can be obtained using FTIR analysis of the products. Major observed IR assignments correspond with $\mathrm{C}=\mathrm{O}$ vibration in the $1750 \mathrm{~cm}^{-1}$ region, different $\mathrm{CH}_{2}$ vibrations in the regions $1150,1250 \mathrm{~cm}^{-1}$, and $\mathrm{CH}_{2} / \mathrm{C}-\mathrm{O}-\mathrm{H}$ vibrations in the $1400 \mathrm{~cm}^{-1}$ region. Similar spectra observed for imprinted and non-imprinted materials seem to show that IR identification of the template in the molecularly imprinted hydrogels is not possible. Besides possible template leakage due to the above described isolation process applied to the products, it should be stressed that the imprinted hydrogels synthesized in this work were obtained with a low ratio of template/functional-monomer, when compared with classical MIP recipes. ${ }^{21}$ Indeed, there is further possibility of recipe optimization, involving the initial amounts of template/functional monomer/crosslinker, aiming at the production of imprinted smart hydrogels combining the ability for stimulation (viscoelasticity) with molecular recognition capabilities (stability of imprinting cavities).

\section{Swelling degree with respect to selective sorption}

The swelling ratio of the hydrogel is the main influencing parameter that has to be controlled when used as stationary phase or carrier in the field of separation science/medical sciences. Having considered this critical parameter the hydrogels swelling degree was monitored with respect to the different analytes including the target molecule 3-APy. Figure 9(a)-(d) depicts the dependence of SR of the FRP and CRP generated hydrogels with respect to its recognition capacity and selectivity for target molecule 3-APy, its structural analogue 4-APy and a random drug, caffeine.

From Fig. 9(a) and (b) it is observed that the imprinted FRP (MIH-FRP) hydrogels showed noticeable differences in SRs with respect to the target molecule and its analogues. On the other hand, NIH-FRP hydrogels did not show differences in SR for all three different molecules tested. This can be explained by the fact that during the imprinting process one could expect generation of high recognition molecular sites in the hydrogel polymer network by self -assembly of T-M. This leads to a high number of tailored stereo-cavity binding sites in the MIH-FRP resulting in high SR values for the target 3-APy. On the other hand the differences in the SR for MIH-FRP between 3-APy and 4-APy are small and can be explained by the molecular volume and resonance structures (Fig. 10(a) and (b)).

As far as molecular volume is considered the volumes of the target and of the structural analogue are similar. Looking at the resonance structures (Fig. 10(a) and (b)) the negative charge present on the para nitrogen in 4-APy increases the basicity of the molecule and thus results in a relatively high SR with respect to 3-APy. The measured pKa value of 4-APy further supports the observed result (Fig. 10). A similar result has been observed by Mullet et al., confirming that the interaction between the $-\mathrm{COOH}$ and $-\mathrm{NH}_{2}$ group becomes stronger with increasing basicity. ${ }^{40}$ These findings foresee the preparation of new MIPs imprinted with a structural analogue (or surrogate). A MIP can be imprinted with a structural analogue (dummy template or surrogate) of similar size and shape 


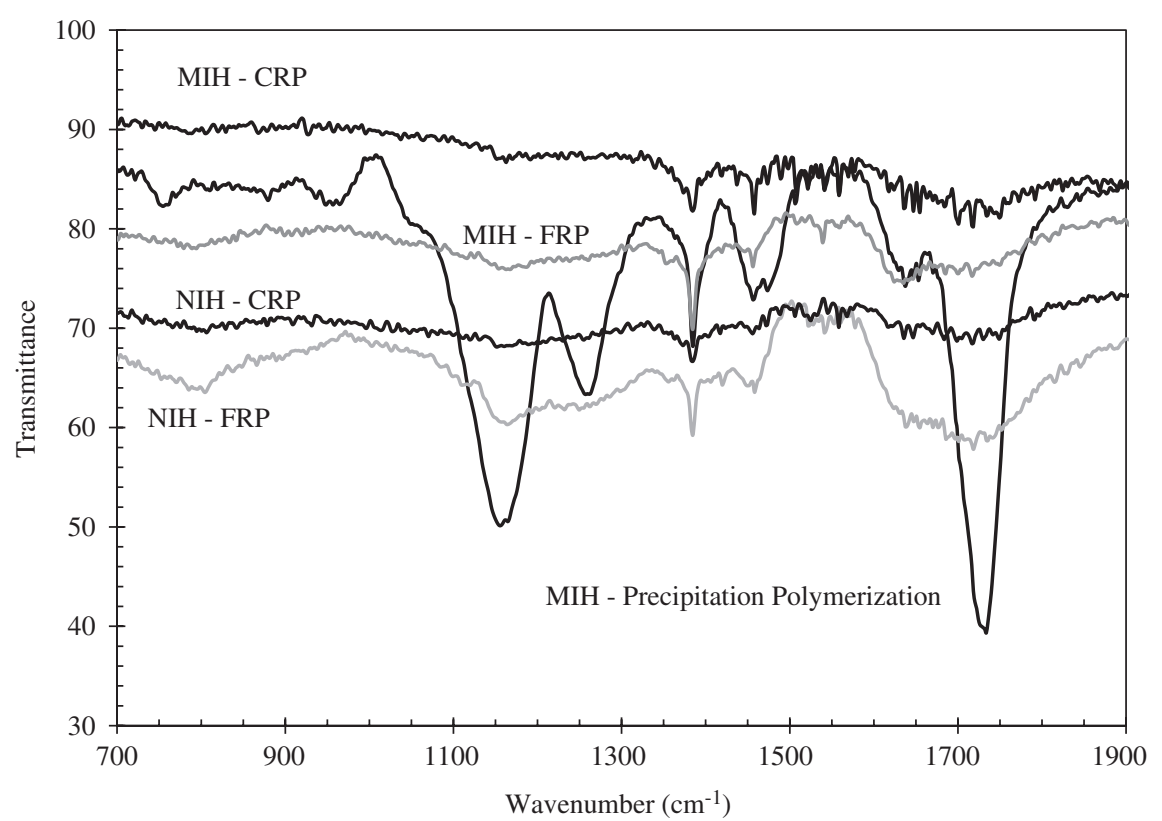

Figure 8. FTIR spectra of imprinted and non-imprinted dried hydrogels produced through FRP and RAFT polymerization. For comparison, the FTIR spectrum of a similar MIH hydrogel soft powder was also included.

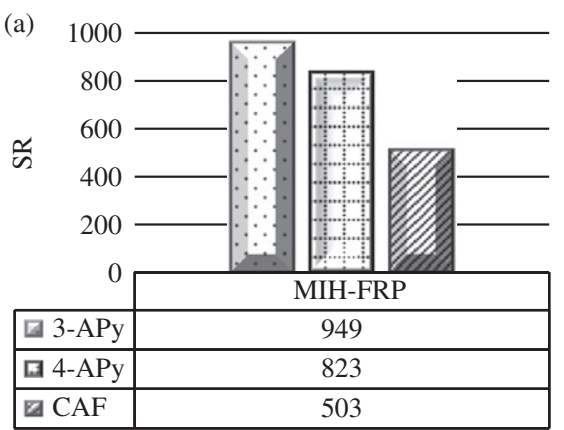

(c)

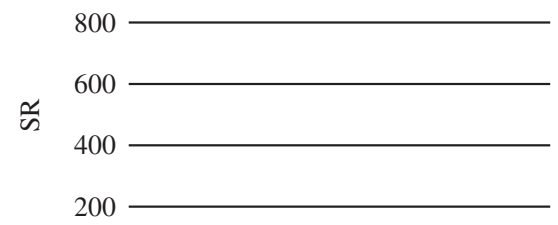

\begin{tabular}{|l|c|}
\cline { 2 - 2 } \multicolumn{1}{c|}{} & \multicolumn{1}{c|}{0} \\
\cline { 2 - 3 } \multicolumn{1}{c|}{} & MIH-CRP \\
\hline 3-Apy & 52 \\
\hline 4-Apy & 64 \\
\hline 界 & 52 \\
\hline
\end{tabular}

(b)

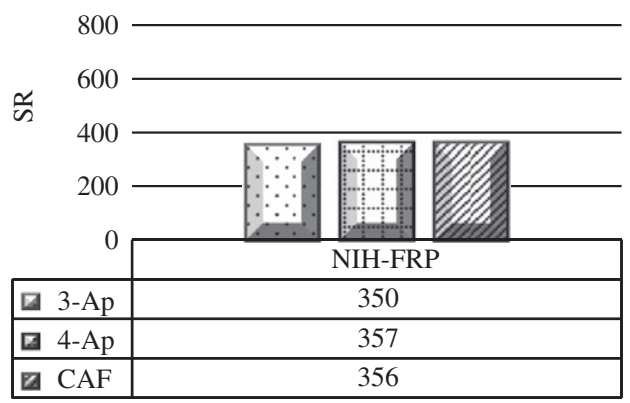

(d)

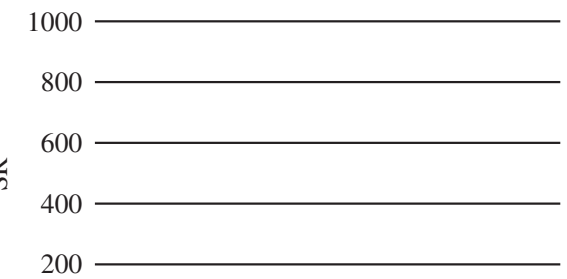

\begin{tabular}{|l|c|}
\multicolumn{1}{c|}{} & \multicolumn{1}{c|}{0} \\
\cline { 2 - 3 } \multicolumn{1}{c|}{} & NIH-CRP \\
\hline 3-py & 36 \\
\hline $\mathbf{a}$ 4-py & 46 \\
\hline 界 CAF & 41 \\
\hline
\end{tabular}

Figure 9. Swelling ratio of the hydrogels in aqueous solutions containing different analytes (3-aminopyridine, 4-aminopyridine and caffeine): (a) MIH-FRP; (b) NIH-FRP; (c). MIH-CRP; (d). NIH-CRP.

to the target molecule but with a lower pKa value. For instance, in this case 3-APy imprinted hydrogels could be used to fetch 2-APy and 4-APy from active pharmaceutical ingredients (API).

However, the remarkable difference observed in SR between 3 -APy and caffeine (2-fold in magnitude) confirms the presence of stereo selective sites in MIH-FRP due to the imprinting process. There is no significant difference in SR of NIH-FRP; over the three molecules studied this behavior implies the existence of physical adsorption rather than molecular recognition. This result further confirms the promise of imprinting in MIH-FRP.

On the other hand, interesting SR data were observed for the imprinted (MIH-CRP) and non-imprinted (NIH-CRP) hydrogels prepared by CRP using RAFT agent. The results are presented in Fig. 9(c) and (d): no substantial difference was noticed in SR between MIH and NIH-CRP. The only plausible explanation would be the influence of RAFT addition. Despite the quantity being very 

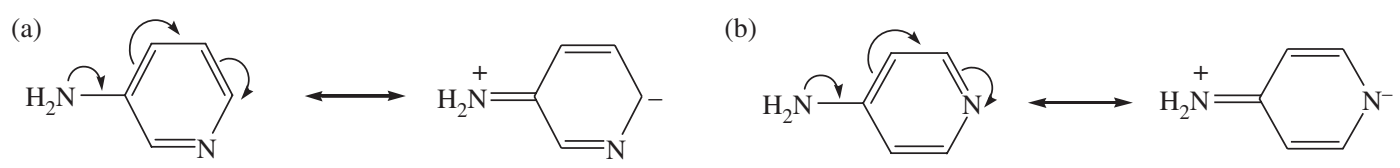

Figure 10. Stable resonance structures of (a) 3-APy (pKa 5.98 ${ }^{39}$ ) and (b) 4-APy (pKa 9.1739).

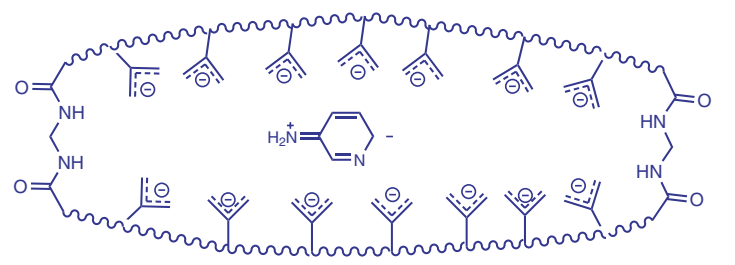

(a) MIH-FRP (homogeneous network)

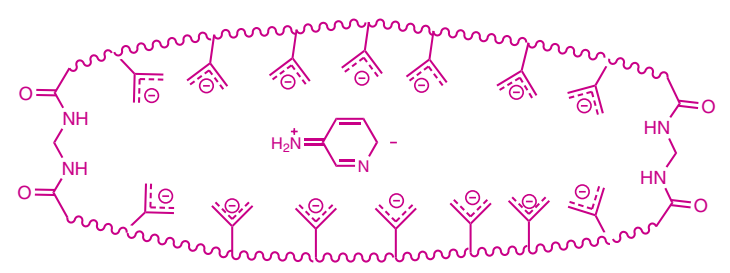

(c) MIH-CRP (homogeneous network)

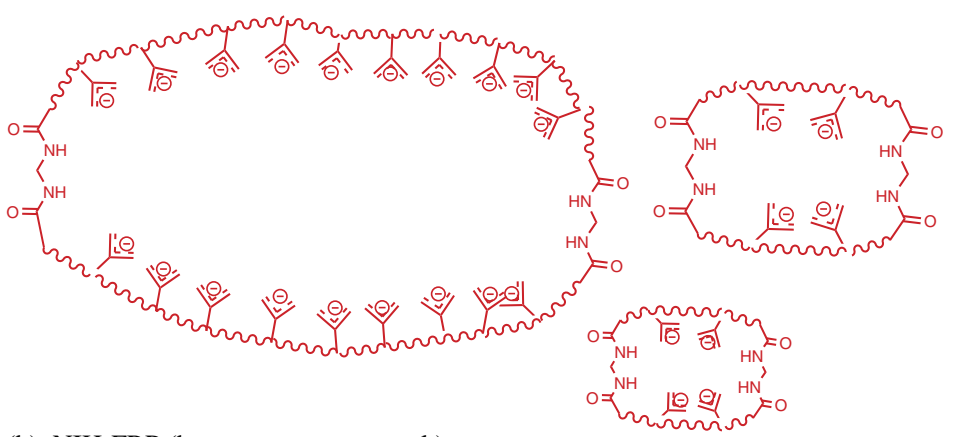

(b) NIH-FRP (heterogeneous network)

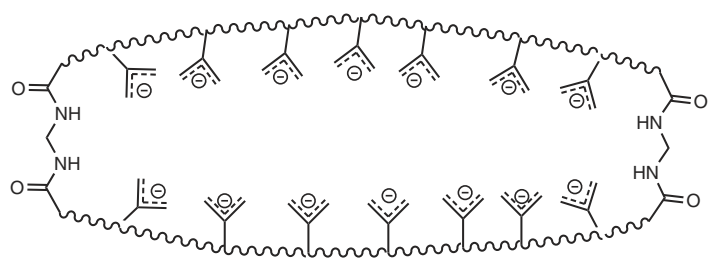

(d) NIH-CRP (homogeneous network)

Figure 11. Expected polymer network cavity formation with FRP and CRP imprinted and non-imprinted hydrogels. Results obtained are consistent with control of the reaction kinetics by the RAFT agent and formation of polymer networks containing homogeneous cavities regardless of the presence of a template.

small $(0.1 \%)$, the rate of the reaction decreased enormously and this would be the reason for observed similar SR values between $\mathrm{MIH}$ and NIH-CRP. The decrease of primary chain length of networks synthesized using CRP ${ }^{17,18}$ is a well-known issue, which also explains the drop in the observed SR values.

The introduction of a RAFT agent is currently used by many researchers ${ }^{9,14-19}$ mainly to obtain a homogeneous polymer network by avoiding the presence of broad distributions of sizes for any sub-structures like sub-chains or loops. One could rationally design a close to ideal polymer network architecture by using RAFT agent. Here in our work we employed the RAFT agent to moderate fast AA (exothermic reaction) polymerization and to reach an optimal gelation time before micro-reactor feeding. The purpose of RAFT addition was successful in optimizing the reaction conditions but the SR towards selectivity MIH-CRP was not up to expectations. Also no significant difference in SR between $\mathrm{MIH} / \mathrm{NIH}-\mathrm{CRP}$ produced particles was observed for the three test molecules studied. This is likely to be explained by the fact that the RAFT agent effectively controlled the reaction kinetics and hence producing the polymer networks with homogeneous cavities regardless of the presence of template (MIH-CRP). MIH-CRP (Fig. 11(c)) and NIH-CRP (Fig. 11(d)) could have cavities of similar size and so that resulted in the observed similar SR values.

In order to obtain some knowledge about the molecular architecture, the soluble fraction of SRSH or their linear counterparts was experimentally measured using a SEC apparatus coupled to a tetra detector array including refractive index, light scattering, viscosity and ultraviolet detection. These measurements are especially important to obtain insights on the network formation dynamics and involved kinetic mechanisms. For this purpose, calibration was conducted using PEO (poly ethylene oxide) standards with known molecular weight: 5300, 66600 and $920000 \mathrm{~g} \mathrm{~mol}^{-1}$ and the approximate molecular weight of the polymer was estimated using the following equation:

$$
M=\alpha \times V_{e}^{\beta} \Longleftrightarrow \log M=\log \alpha+\beta \times \log V_{e}
$$

where, $M$ is the molecular weight of the polymers $\left(\mathrm{g} \mathrm{mol}^{-1}\right), \alpha$ is the intercept of the calibration curve, $V_{e}$ is retention volume and $\beta$ the slope of the calibration curve. Calibration was necessary because of the injection of high viscosity soluble polymer fraction which would limit passage through the capillaries resulting in the failure of absolute value detection.

Tetra detector SEC chromatograms (UV absorbance) obtained for the FRP and CRP linear polymer are shown in Fig. 12(a) and (b). From the graph one can clearly see that the FRP-produced network hydrogel analogues have a wide molecular weight range (from $2 \times 10^{5}$ to $2 \times 10^{9} \mathrm{~g} \mathrm{~mol}^{-1}$ ) whereas the CRP-produced linear polymers are more homogeneous and present lower chain lengths (from $2 \times 10^{5}$ to $8 \times 10^{7} \mathrm{~g} \mathrm{~mol}^{-1}$ and with maximum intensity at $\left.6 \times 10^{5} \mathrm{~g} \mathrm{~mol}^{-1}\right)$. The influence of RAFT agent in producing more homogeneous soluble polymers and networks was further confirmed from the results obtained for the hydrogels produced in the CRP batch and micro-reactor (CRP MRP) polymers (Fig. 12(b)).

The SEC experimental results point to the formation of a rather homogeneous network regardless of the presence or absence of a target molecule and hence generate more non-specific 

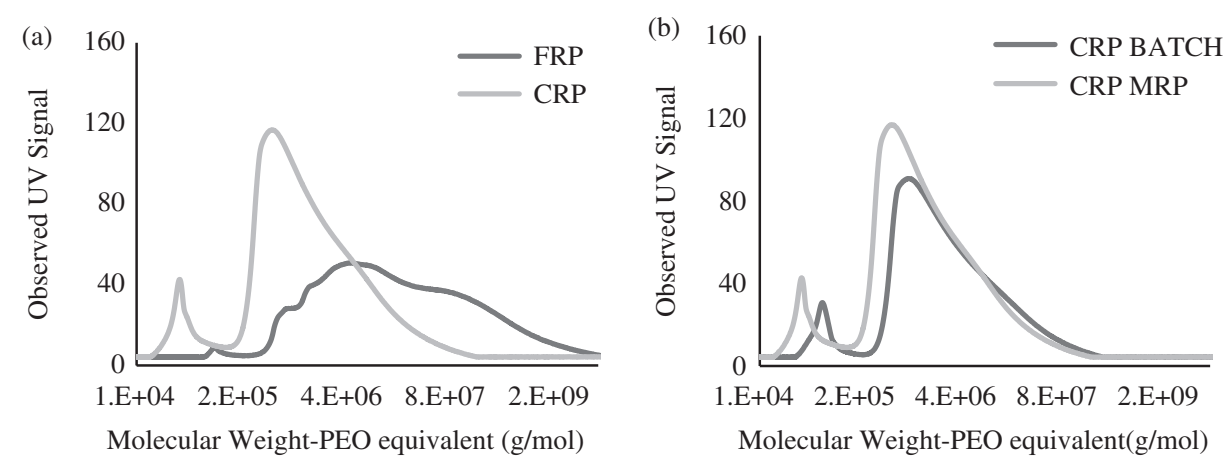

Figure 12. Characterization by SEC with tetra-detection of linear AA polymer. (a) Comparison of FRP and CRP. (b) Comparison of CRP in batch and continuous micro-reactor.
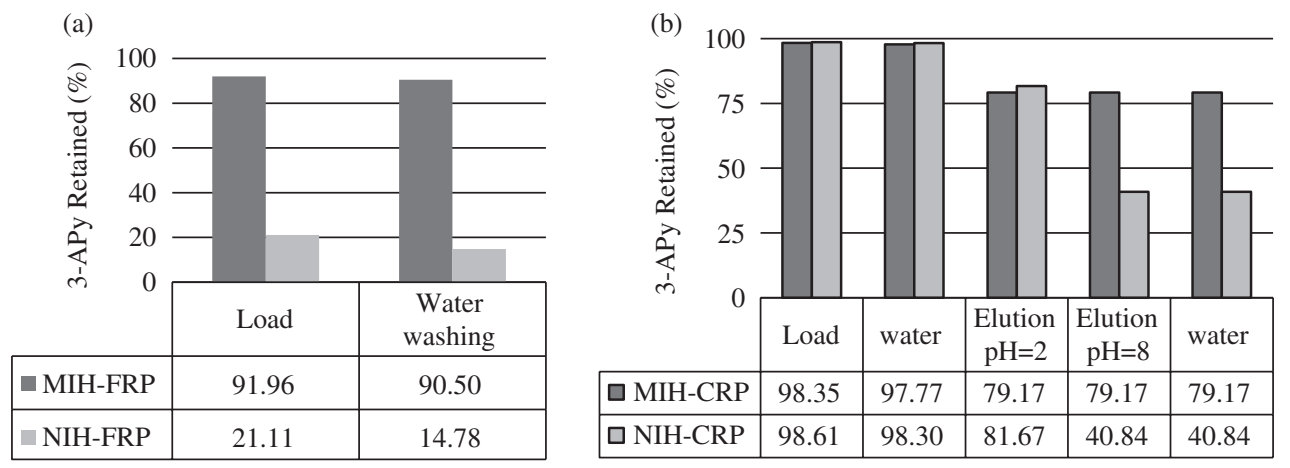

Figure 13. Solid phase extraction experiments involving the different hydrogels produced and 3APy: SPE result of (a) MIH/NIH-FRP; (b) MIH/NIH-CRP hydrogels.

binding sites with uniformly sized cavities which dominate physical adsorption rather than the selective molecular recognition.

\section{Solid phase extraction experiments}

Followed by a swelling test, SPE measurements were carried out in order to obtain knowledge about the retention capacity of $\mathrm{MIH}$ and NIH produced by FRP and CRP.

Results regarding the percentage 3-APy retained in the SPE cartridge for the four different hydrogels studied are presented in Fig. 13(a) (MIH/NIH-FRP), and (b) (MIH/NIH-CRP). FRP-produced hydrogel showed significant differences in the percentage retained between $\mathrm{MIH}$ and $\mathrm{NIH}$ from loading $\left(5 \mathrm{mmol} \mathrm{L}^{-1} 3-\mathrm{APy}\right.$ in aqueous solution). About $100 \%$ of 3-APy was retained in $\mathrm{MIH}$ and $20 \%$ in $\mathrm{NIH}$, showing a promising imprinting effect in MIH hydrogel. Further washing with water did not influence any change in the adsorption amount in $\mathrm{MIH}$ whereas a decrease of about $30 \%$ from the loading test in the case of $\mathrm{NIH}$ (14.78\% retained) showed the existence of physical sorption in the $\mathrm{NIH}$. In contrast the CRP-generated $\mathrm{MIH}$ and $\mathrm{NIH}$ did not show any noteworthy difference in the percentage 3-APy retained (c.100\%). This can be explained again by the fact that in CRP-produced particles physical adsorption is dominant due to the generation of homogeneous cavities by the addition of RAFT agent, as was tentatively explained in the previous section. Further, the existence of physical sorption was proved by elution experiments. After loading and washing, elution steps were carried out with varying $\mathrm{pH}$ solutions. There was no significant liberation of 3-APy after addition of $\mathrm{pH} 2$ solution, whereas $\mathrm{pH} 8$ solution eluted half the retained 3-APy in $\mathrm{NIH}$ and at the same time did not change any retained percentage in $\mathrm{MIH}$, showing that there was also molecular recognition combined with non-specific or physical adsorption.
Although the MIH/NIH-CRP did not show any difference in percentage 3-APy retained by SPE loading, the overall results with elution experiments confirmed the existence of imprinted sites in $\mathrm{MIHs}$.

Lower differences were observed when comparing the performance of batch (bulky materials such as FRP batch hydrogels) and micro-reactor generated CRP SRSH. Swelling is controlled by the primary chain length, which should be similar for batch and micro-reactor RAFT synthesized hydrogels (as showed in Fig. 12(b)). Significant differences between these products should eventually be observed for the kinetics of mass transfer during drug adsorption/desorption processes due to the effect of product morphology.

\section{Frontal analysis}

In order to obtain higher precision data on adsorption/desorption (saturation/release) drug mechanisms, a frontal analysis experiment was considered for characterization of the different smart hydrogels synthesized. As described in the experimental part, adsorption and desorption were monitored in-line through UV absorption for the analytes 3-APy and CAF in NIH-FRP and the resulting chromatograms are illustrated in Fig. 14.

It was observed that the adsorption capability with respect to retention volume of the NIH-FRP hydrogels for 3-APy was at least 2 -fold higher in comparison with caffeine (c. $30 \mathrm{~mL}$ retention volume for 3-APy and c. $16 \mathrm{~mL}$ for CAF). The higher volume indicates the high affinity for 3-aminopyridine due to acid-base interactions of acrylic acid with 3-APy. This is unlikely to occur with caffeine which has the lower saturation retention volume of $c .16 \mathrm{~mL}$. After loading, 3-APy and CAF release profiles were monitored using water as an eluent and the liberation profile of the analytes are 
(a)

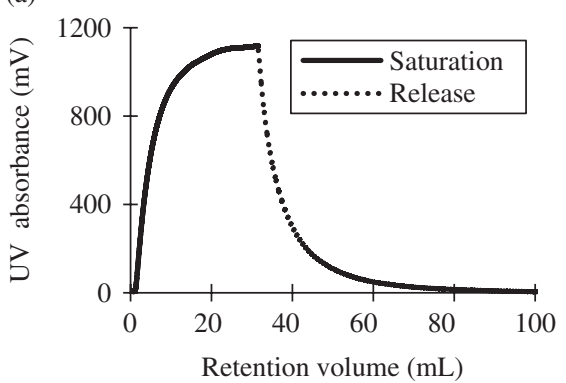

(b)

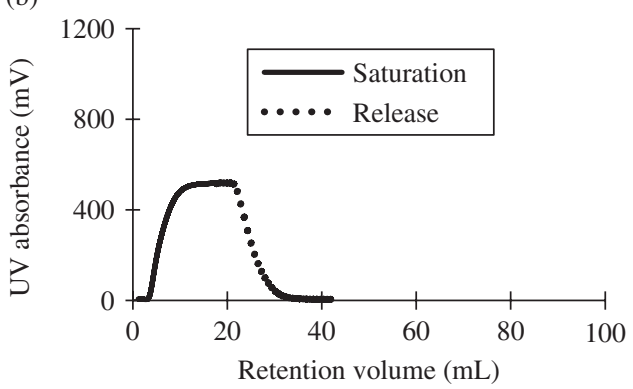

Figure 14. Saturation and release profiles for (a) 3-APy and (b) CAF in an AA based non-imprinted SRSH. Frontal analysis conditions: $C_{0}=0.5 \mathrm{mmol} \mathrm{L}^{-1}$,

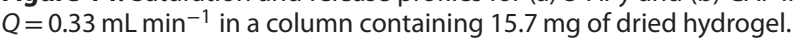

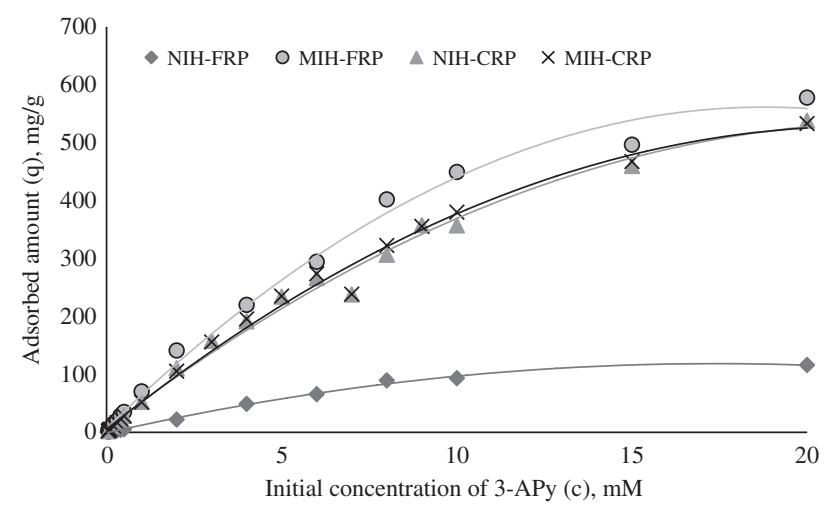

Figure 15. Equilibrium binding isotherms of 3-APy for $\mathrm{MIH} / \mathrm{NIH}-\mathrm{FRP}$ and $\mathrm{MIH} / \mathrm{NIH}-\mathrm{CRP}$ hydrogels.

shown in Fig. 14(a) and (b). The release was faster for CAF than for 3-Apy. This behavior can again be explained as due to the presence of weaker acid base interaction than that between AA and 3-Apy (Fig. 14(a)).

It is important to mention here that although frontal analysis is an accurate method to determine the saturation capacity of the analytes per mass of hydrogel, the experimental challenges prevent its use due to the high swelling of the imprinted hydrogels leading to the maximum pressure limit being reached. Hence, to obtain adsorption capacities for the imprinted hydrogels batch rebinding tests were conducted. Design of new formulations (e.g. considering the ratio RAFT agent/monomer to control the swelling ratio) with generation of materials with good conditions for column packing should hopefully allow study of the kinetics of mass transfer for drug adsorption/desorption processes using a multi-step frontal analysis method.

\section{Batch adsorption test (FRP vs. CRP)}

Difficulties faced in the frontal analysis resulted in the use of batch rebinding experiments to quantify the amount of 3-APy adsorbed $\left(q \mathrm{mg} \mathrm{g}^{-1}\right)$ in $\mathrm{MIHs}$ and NIHs. The results obtained are shown in Fig. 15.

It can be clearly seen from Fig. 15 that the amount adsorbed, $q$ in MIH-FRP is remarkably higher than $q$ in NIH-FRP. The estimated quantity of 3-APy adsorbed in aqueous solution for MIH-FRP is 8 -fold higher in magnitude (c. $600 \mathrm{mg} \mathrm{g}^{-1}$ ) when compared with NIH-FRP (c. $75 \mathrm{mg} \mathrm{g}^{-1}$ ). The outstanding $q$ value observed for MIH-FRP hydrogel is strong evidence of the existence of a large amount of recognition cavities generated by T-M self-assembly. On the other hand similar $q$ values estimated for $\mathrm{MIH} / \mathrm{NIH}-\mathrm{CRP}$ (c. $530 \mathrm{mg} \mathrm{g}^{-1}$ ) showed the dominance of non-specific acid-base interaction over selective recognition sites generated by the imprinting molecules in MIH-CRP. As explained earlier RAFT agent used in CRP totally controlled the polymer architecture in generating uniform cavities regardless of the presence of template (MIH-CRP) or its absence (NIH-CRP) and hence favors the non-specific interactions.

\section{Comparison of current results with published work}

Table 2 shows the work done by other researchers in which aminopyridine was considered the target in the generation of bulk classical imprints (MIPs), MIPs microspheres and MIPs hybrid with NIPA hydrogel. To our best knowledge none carried out research in synthesizing smart hydrogels microparticles integrating CRP and imprinting processes for the best possible outcome. The highlight of this current research is starting from synthesis to end product analysis, water was used as solvent, whereas other researchers have quoted the use of dimethylformamide (DMF), chloroform $\left(\mathrm{CHCl}_{3}\right)$ and acetonitrile $(\mathrm{MeCN})$ as working solvents ${ }^{38,41-43}$ (Table 2).

Regarding SPE results the SRSH produced in this current research showed a better response than that presented by Rustem et al. ${ }^{41}$ and Cummins et $a .^{43}$ The adsorption capacity $q$ of MIHs synthesized $\left(c .6000 \mathrm{mmol} \mathrm{kg}^{-1}\right)$ in this current work is 1000 times higher than $q$ quoted by Liu Xueyong et al. ${ }^{42}$ (c. $6 \mathrm{mmol} \mathrm{kg}^{-1}$ ). Due to the experimental difficulties caused by the enormous swelling we were not able to determine the capacity factor to compare with the results of Lai et al. ${ }^{38}$ (Table 2 ).

\section{CONCLUSIONS}

A feasibility analysis of the production of RAFT imprinted smart hydrogel particles in a customised continuous flow micro-reactor was the main goal of this research work. Importing the tailor-made sites into the hydrogel network in aqueous media and tackling the huge drop in crosslinking level (from $80 \%$ in classical MIPs to $1 \%$ in hydrogels) were the most important challenges faced in this work. However, imprinted materials were successfully prepared both in batch mode and a continuous micro-reactor. It was shown to be possible to produce smart hydrogel particles with fairly regular size and shape in a continuous micro-reactor, contrasting with the bulky materials obtained with classical batch processes (with FRP or (RP). An extension of this research should include new developments on the design of continuous micro-reactor operating conditions (temperature, composition, residence time, tubing diameter, etc.), allowing the production of molecularly imprinted organic particles with special shapes and morphologies that are difficult to 
obtain in batch processes (e.g. hollow molecularly imprinted particles for drug delivery carriers).

The addition of RAFT agent in CRP helped in optimising the experimental conditions of the continuous microfluidic approach. Experimental results proved that molecular imprints generated by FRP and CRP showed outstanding performance in adsorption behaviour, and the $q$ value estimated was found to be about 1000 times higher than the value presented by other researchers in comparable chemical systems. SPE results further evidenced the promise of imprinting in MIH-FRP/CRP.

The results show that we have obtained a new class of regular shaped (oval) functional material with controlled swelling and capable of holding a large amount of analyte. Liberation of the analyte can be triggered by an environmental change. However, we are yet to assess the impact of RAFT agent on the molecular imprinting process. Further research exploring a change to the initial ratio RAFT agent/monomer (therefore establishing the primary chain length of the network at specified crosslinker content) and demonstrating the impact of other RAFT agents could be the next step in this thread.

\section{ACKNOWLEDGEMENTS}

The authors thank FCT and FEDER under Programme COMPETE (Project PEst-C/EQB/LA0020/2013), QREN, ON2 and FEDER (Project NORTE-07-0162-FEDER-000050) and QREN, ON2 and FEDER (Project NORTE-07-0124-FEDER- 0000014 - Polymer Reaction Engineering). P.K. thanks NORTE-07-0124-FEDER-0000014 for the Post Doctoral grant.

\section{REFERENCES}

1 Abdurrahmanoglu S and Okay O, Preparation of homogeneous hydrogels by controlling the crosslinker reactivity and availability. J Macromol Sci A 45:769-775 (2008).

2 Zhang Q, Su K, Chan-Park MB, Wu H, Wang D and Xu R, Development of high refractive ZnS/PVP/PDMAA hydrogel nanocomposites for artificial cornea implants. Acta Biomater 10:1167-1176 (2014). RT, Choy WM, Cao H, Qattan I, Chiao JC, Ip WY et al., Fabrication and characterization of biomimetic multichanneled crosslinked-urethane-doped polyester tissue engineered nerve guides. J Biomed Mater Res A 102:2793-2804 (2014).

4 Sharma B, Fermanian S, Gibson M, Unterman S, Herzka DA, Cascio B et al., Human cartilage repair with a photoreactive adhesive hydrogel composite. Sci Translational Med 5:167 (2013).

5 Galaev I and Mattiasson B, Smart Polymers. Applications in Biotechnology and Biomedicine, 2 nd edition. CRC Press (2007).

6 Bajpai A, Shukla S, Saini R and Tiwari A, Stimuli Responsive Drug Delivery Systems: From Introduction to Application. Smithers Rapra Technology (2010).

7 Islam MR, Lu Z, Li X, Sarker AK, Hu L, Choi P et al., Responsive polymers for analytical applications: a review. Anal Chim Acta 789:17-32 (2013).

8 Ozdil D and Aydin HM, Polymers for medical and tissue engineering applications. J Chem Technol Biotechnol 89:1793-1810 (2014).

9 Diehl C, Laurino P, Azzouz N and Seeberger PH, Accelerated continuous flow RAFT polymerization. Macromolecules 43:10311-10314 (2010).

10 Lorber N, Pavageau B and Mignard E, Investigating acrylic acid polymerization by using a droplet/based millifluidics approach. Macromol Symp 296:203-209 (2010).

11 Yadav AK, de la Cal JC and Barandiaran MJ, Feasibility of tubular microreactors for emulsion polymerization. Macromol React Eng 5:69-77 (2011).

12 Wan J and Stone HA, Coated gas bubbles for the continuous synthesis of hollow inorganic particles. Langmuir 28:37-41 (2012).

13 Khan IU, Serra CA, Anton N and Vandamme T, Microfluidics: a focus on improved cancer targeted drug delivery systems. J Control Release 172:1065-1074 (2013). 
14 Chaduc I, Crepet A, Boyron O, Charleux B, Agosto FD and Lansalot $\mathrm{M}$, Effect of the $\mathrm{pH}$ on the RAFT polymerization of acrylic acid in water. Application to the synthesis of poly(acrylic acid)-stabilized polystyrene particles by RAFT emulsion polymerization. Macromolecules 45:6013-6023 (2012).

15 Gonçalves MAD, Pinto VD, Costa RAS, Dias RCS, Hernándes-Ortiz JC and Costa MRPFN, Stimuli-responsive hydrogels synthesis using free radical and RAFT polymerization. Macromol Symp 333:41-54 (2013).

16 Gonçalves MAD, Pinto VD, Costa RAS, Dias RCS, Hernándes-Ortiz JC and Costa MRPFN, Dynamics of network formation in aqueous suspension RAFT styrene/divinylbenzene copolymerization. Macromol Symp 333:273-285 (2013).

17 Moad G, RAFT (reversible addition-fragmentation chain transfer) crosslinking (co)polymerization of multi-olefinic monomers to form polymer networks. Polym Int 64:15-24 (2015).

18 Matyjaszewski $\mathrm{K}$ and Spanswick J, Controlled/living radical polymerization. Mater Today 8:26-33 (2005).

19 Moad G, Rizzardo E and Thang SH, Living radical polymerization by the RAFT process - a third update. Aust J Chem 65:985-1076 (2012).

20 Kadhirvel $P$, Azenha M, Schillinger E, Halhalli MR, Silva AF and Sellergren $B$, Recognitive nano-thin-film composite beads for the enantiomeric resolution of the metastatic breast cancer drug aminoglutethimide. J Chromatogr A 1358:93-101 (2014).

21 Sellergren B, Molecularly Imprinted Polymers. Man-Made Mimics of Antibodies and their Applications in Analytical Chemistry. Elsevier, 213 pp. (2001).

22 Mosbach K, Molecular imprinting. Trends Biochem Sci 19:9-14 (1994).

23 Shokat KM, Leumann CJ, Sugasawara R and Schultz PG, A new strategy for the generation of catalytic antibodies. Nature $\mathbf{3 3 8}$ (6212):269-271 (1989).

24 Li S, Ge Y and Turner APF, A catalytic and positively thermosensitive molecularly imprinted polymer. Adv Funct Mater 21:1194-1200 (2011).

25 Zhang H, Water-compatible molecularly imprinted polymers: promising synthetic substitutes for biological receptors. Polymer 55:699-714 (2014).

26 Zhang H, Controlled/'living' radical precipitation polymerization: a versatile polymerization technique for advanced functional polymers. Eur Polym J 49:579-600 (2013).

27 Zhou T, Jørgensen L, Mattebjerg MA, Chronakis loannis IS and $\mathrm{Ye} \mathrm{L}$, Molecularly imprinted polymer beads for nicotine recognition prepared by RAFT precipitation polymerization: a step forward towards multi-functionalities. RSC Adv 4: 30292-30299 (2014).

28 Pan G, Ma Y, Zhang Y, Guo X, Li C and Zhang H, Controlled synthesis of water-compatible molecularly imprinted polymer microspheres with ultrathin hydrophilic polymer shells via surface-initiated reversible addition-fragmentation chain transfer polymerization. Soft Matter 7: 8428-8439 (2011).

29 Ye L and Mosbach K, Molecular imprinting: synthetic materials as substitutes for biological antibodies and receptors. Chem Mater 20:859-868 (2008).

30 Kadhirvel P, Dias RCS and Costa MRPFN, Microreactor generated RAFT imprinted smart hydrogels, in 3rd International Symposium on Controlled/Living Polymerization: From Synthesis to Application, Antalya,
Turkey, Book of Abstracts:150-151. [Online]. (2014). Available: https://bibliotecadigital.ipb.pt/bitstream/10198/9479/3/AbstractC LP14Book.pdf [10 March 2015].

31 Machado C, Freitas A, Kadhirvel P, Dias RCS and Costa MRPFN, Production of RAFT Imprinted Smart Hydrogel Particles in a Continuous Flow Micro-Reactor, in 12th International Chemical and Biological Engineering Conference, Porto, Portugal, Book of Extended Abstracts: 5-20-5-22. [Online]. (2014). Available: https://bibliotecadigital. ipb.pt/bitstream/10198/10429/1/Chempor2014-A.pdf [10 March 2015].

32 Machado C, Oliveira T, Reitor P, Oliveira D, Freitas A, Kadhirvel P, Dias RCS and Costa MRPFN, Development of Tailored Hydrogels using RAFT Polymerization in Continuous Flow Microreactor, in 8th ECNP International Conference on Nanostructured Polymers and Nanocomposites, Dresden, Germany, Collection of Abstracts:O16. [Online]. (2014). Available: https://biblioteca digital.ipb.pt/bitstream/10198/10480/1/Dresden_A.pdf [10 March 2015].

33 Guiochon G and Katti AM, Fundamentals of Preparative and Nonlinear Chromatography. Academic Press, Boston (1994).

34 Quinones I and Guiochon G, Extension of a Jovanovic-Freundlich isotherm model to multicomponent adsorption on heterogeneous surfaces. J Chromatogr A 796:15-40 (1998).

35 Kadhirvel P, Azenha M, Shinde S, Schillinger E, Gomes P, Sellergren B et al., Imidazolium-based functional monomers for the imprinting of the anti-inflammatory drug naproxen: comparison of acrylic and sol-gel approaches. J Chromatogr A 1314:115-123 (2013).

36 Svenson J, Andersson HS, Piletsky SA and Nicolls IA, Spectroscopic studies of the molecular imprinting self-assembly process. $J \mathrm{Mol}$ Recognit 11:83-86 (1998).

37 Andersson HS and Nicholls IA, Spectroscopic evaluation of molecularly imprinted polymerization systems. Bioorgan Chem 25:203-211(1997).

38 Lai J-P, Lu X-Y, Lu C-H, Ju H-F and He X-W, Preparation and evaluation of molecularly imprinted polymeric microspheres by aqueous suspension polymerization for use as a high performance liquid chromatography stationary phase. Analytica Chimica Acta 442:105-111 (2001).

39 Caballero NA, Melendez FJ, Munoz-Caro C and Nino A, Theoretical prediction of relative and absolute pKa values of aminopyridines. Biophys Chem 124:155-160 (2006).

40 Mullett WM, Dirie MF, Lai EPC, Guo HS and He XW, A 2-aminopyridine molecularly imprinted polymer surrogate micro-column for selective solidphase extraction and determination of 4-aminopyridine. Anal Chim Acta 414:123-131 (2000).

41 Kecili R, Billing J, Nivhede D, Sellergren B, Rees A and Yilmaz F, Fast identification of selective resins for removal of genotoxic aminopyridine impurities via screening of molecularly imprinted polymer libraries. J Chromatogr A 1339:65-72 (2014).

42 Liu XY, Zhou T, Du ZW, Wei Z and Zhang JH, Recognition ability of temperature responsive molecularly imprinted polymer hydrogels. Soft Matter 7:1986-1993 (2011).

43 Cummins W, Duggan P and McLoughlin P, Systematic cross-selectivity study of the factors influencing template receptor interactions in molecularly imprinted nitrogen heterocycles. Biosens Bioelectro 22:372-380 (2006). 